\title{
Recent Advances in Spectroscopic Gas Sensing With Micro/Nano-Structured Optical Fibers
}

\author{
Wei JIN ${ }^{1,2 *}$, Haihong BAO ${ }^{1,2}$, Pengcheng $\mathrm{ZHAO}^{1,2}$, Yan $\mathrm{ZHAO}^{1,2}$, Yun $\mathrm{QI}^{1,2}$, \\ Chao WANG ${ }^{1,2}$, and Hoi Lut $\mathrm{HO}^{1,2}$ \\ ${ }^{1}$ Department of Electrical Engineering, The Hong Kong Polytechnic University, Hong Kong, China \\ ${ }^{2}$ The Hong Kong Polytechnic University Shenzhen Research Institute, Shenzhen 518057, China \\ *Corresponding author: Wei JIN_Ｅ-mail: eewjin@polyu.edu.hk
}

\begin{abstract}
With micro- and nano-structured optical fibers, parts-per-million to parts-per-trillion level gas detection has been demonstrated for a range of gases such as methane, acetylene, ethane, carbon monoxide, hydrogen, and oxygen. We review the recent development in optical fiber gas cells and gas detection systems based on direct absorption, photothermal, photoacoustic, and stimulated Raman spectroscopies.
\end{abstract}

Keywords: Gas sensor; optical fiber sensor; micro-structured optical fiber; nanofiber; laser spectroscopy

Citation: Wei JIN, Haihong BAO, Pengcheng ZHAO, Yan ZHAO, Yun QI, Chao WANG, et al., "Recent Advances in Spectroscopic Gas Sensing With Micro/Nano-Structured Optical Fibers," Photonic Sensors, 2021, 11(2): 141-157.

\section{Introduction}

Gas detection has increasingly important application in environmental monitoring, industrial safety protection, medical instruments, etc. Among many different technologies of gas sensing, laser spectroscopy that makes use of the "finger-print" spectral features of gas molecules offers unique advantage in specificity [1]. Traditional free-space spectroscopic systems employ discrete optical components, which have limitations in size and long-term stability, and require precision alignment during construction. The use of optical fibers for light transmission simplifies the optical alignment and enables flexible systems as well as remote detection capability [2]. However, the sensing elements, which are the platforms of light-gas interaction, are mostly bulky free space gas cells.

Attempt has been made to construct all-optical-fiber gas cells [3, 4], which enable compact sensing elements as well as easy integration into optical fiber systems. D-shaped optical fibers were studied for gas detection by exploiting the evanescent field extending into the surrounding air. However, the sensitivity is low due to the very small fraction of evanescent field in air [3].

Recent development of micro- and nanostructured optical fibers (MNOFs) opens the possibility of constructing all-fiber gas cells for high sensitivity sensing [5-9]. MNOFs are typically single material (silica) fibers with air-holes running along the length of the fiber. They can be designed to support a propagation mode with significant amount of mode power in air, enabling efficient light-gas interaction in the fiber over a long distance. The mode field area of an MNOF is much smaller than that of a typical free space beam, which would enhance the sensitivity of non-linear spectroscopic

Received: 2 March 2021 / Revised: 19 March 2021

(C) The Author(s) 2021. This article is published with open access at Springerlink.com

DOI: $10.1007 / \mathrm{s} 13320-021-0627-4$

Article type: Review 
sensors. In addition, MONFs have novel optical, thermal, and acoustic properties, which could enable new sensing concepts.

In this article, we review recent progress in the development of MNOF gas cells and all-fiber gas sensing systems based on direct absorption, photothermal, photoacoustic and Raman spectroscopy principles, and discuss possible future research directions.

\section{MNOF gas cells}

An optical fiber gas cell should allow the maximal overlap of its mode field with the gas sample. Two broad categories of MNOFs may be used for this purpose: micro-structured hollow-core fibers (HCFs) that support a light mode in the air-core [10-13] and nanoscale solid-core fibers that have a significant fraction of evanescent field in the surrounding air $[8,9]$.

\subsection{Micro-structured HCF gas cells}

Figure 1 shows two types of micro-structured HCFs that may be used for gas cells. They are photonic-bandgap (PBG) HCFs and anti-resonant (AR) HCFs. A PBG-HCF transmits light over a narrow spectral range, from tens to a few hundred nanometers, determined by the PBG of the periodic air-silica cladding [5]. The central wavelength of the transmission band can be designed to be from the visible to the near infrared, and the propagation loss demonstrated ranges from $\sim 1 \mathrm{~dB} / \mathrm{m}$ in the visible to $\sim 10 \mathrm{~dB} / \mathrm{km}$ around $2 \mu \mathrm{m}$ [10-12]. A PBG-HCF can be bent down to a few centimeters in diameter without introducing significant loss [11]. An AR-HCF guides light via the "anti-resonant" and "prohibited-coupling" principles, which typically has much broader transmission bands $[6,7]$. The propagation loss of AR-HCFs has been reduced significantly recently to the level comparable to silica single mode fibers (SMFs) between $600 \mathrm{~nm}$ and $1100 \mathrm{~nm} \mathrm{[13]} \mathrm{and} 0.28 \mathrm{~dB} / \mathrm{km}$ from $1500 \mathrm{~nm}-$ $1600 \mathrm{~nm}$ [14] by use of a nested anti-resonant node-less fiber (NANF) structure as shown in
Fig. 1(c). Comparing with PBG-HCFs, the current AR-HCFs are still quite sensitive to bending.

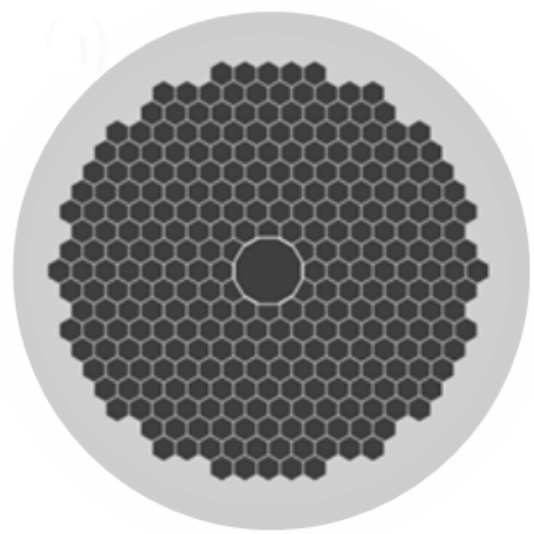

(a)

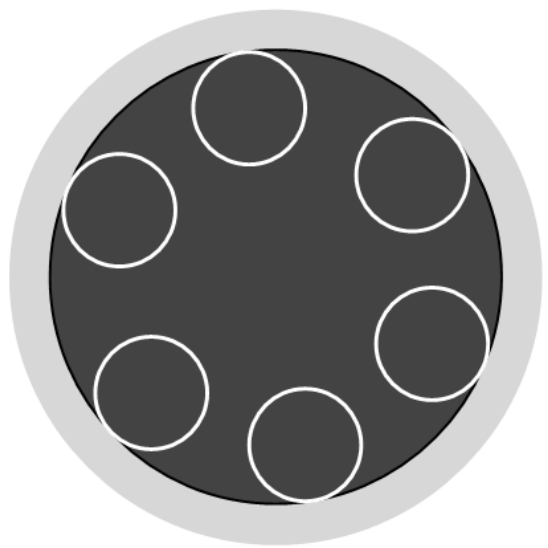

(b)

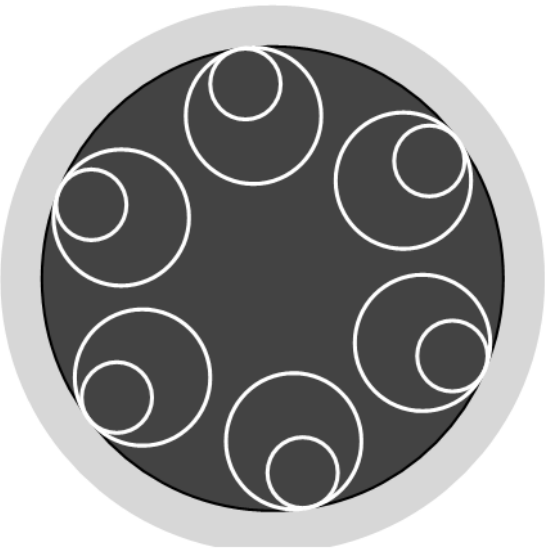

(c)

Fig. 1 Cross-sections of (a) a PBG-HCF, (b) a single ring node-less AR-HCF, and (c) a nested node-less AR HCF or NANF.

An HCF can confine a propagating light mode and a gas sample simultaneously in the hollow-core, and light-sample overlapping approaches $100 \%$. The length of an HCF gas cell can be from centimeters to 
tens of meters, and highly sensitive and yet compact gas sensors could be developed by coiling a length of the HCF into small loops. Gas filling into the hollow-core would have negligible effect on the light confinement and the modal properties of the HCFs [15-17] since most gas species have refractive indexes very close to 1 .

Light coupling into an HCF may be achieved by using free-space optics. Here we are interested in all-fiber gas cells made by splicing the HCF to silica SMF pigtails [17-21]. Splicing avoids the use of discrete optical components, simplifies the optical alignments, improves mechanical stability, and makes it more convenient to integrate with fiber-optic circuits. Figure 2 shows two types of all-fiber gas cells made by fusion splicing and mechanical splicing an HCF with SMFs.

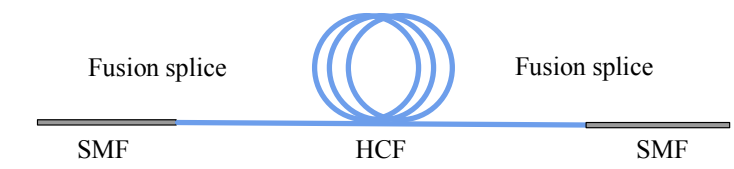

(a)

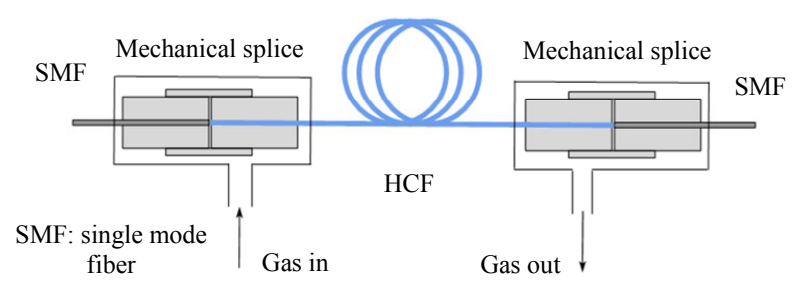

(b)

Fig. 2 All-fiber gas cells made by (a) fusion splicing and (b) mechanical splicing an HCF with SMFs.

An important issue related to the use of long HCF gas cells is the time needed to fill a gas sample into the hollow-core. For gas cells made of mechanical splicing [Fig. 2(b)], gas filling may be achieved via the ends of the HCF through the air gaps at the SMF-HCF joints. However, under free-diffusion condition, it would take hours to fill a 1-meter-long HCF [22]. Pressurization speeds up the gas filling [23], however it increases the system size and complexity. An alternative is to fabricate side-holes or micro-channels along the HCF and to fill gas into the hollow-core via these micro-channels [24-27]. Figure 3 illustrates the cross-sections of a PBG-HCF [28] and an AR-HCF [29] with a micro-channel drilled by use of femtosecond laser micro-machining. With optimized fabrication parameters, hundreds of high-quality micro-channels have been made along a PBG-HCF with average loss of less than $0.01 \mathrm{~dB}$ per channel [28]. The small loss is a result of very small fraction of light field near and within the micro-structured air-silica cladding. For AR-HCFs, the additional loss due to introduction of micro-channels or even a long lateral cut on the outer silica tube is negligible, since the outer tube is not part of the light-guiding structure [30].

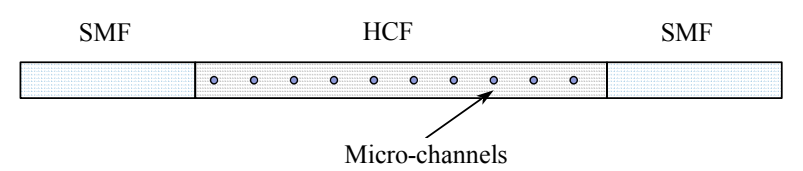

(a)

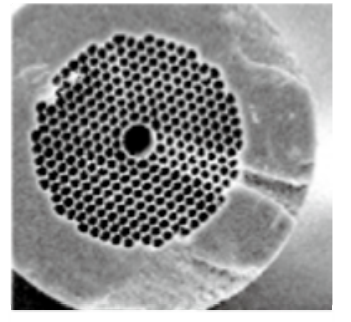

(b)

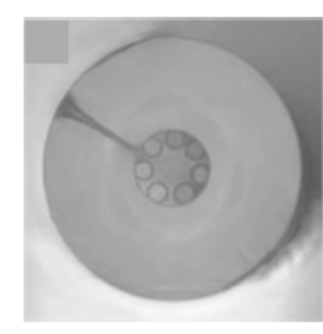

(c)
Fig. 3 HCF gas cell with micro-channels: (a) side view of the gas cell, (b) a PBG-HCF [28], and (c) an AR-HCF [29].

\subsection{Nanoscale solid-core fiber gas cells}

A thin-silica-core with air-clad guides a light mode with a significant amount of evanescent field in air. Two examples of such gas cells are a nano-fiber (NF) sealed within a small-bore tube [31] and a suspended core fiber (SCF) $[9,32]$. An NF may be tapered drawn from a standard SMF by using the "flame-brushing" technique [33], and the diameter of the tapered waist region can be as small as $500 \mathrm{~nm}$ with length up to $10 \mathrm{~cm}$. Figure 4(a) depicts a tapered NF, illustrating the significant amount of light field in air and the much smaller mode field area as compared with the SMF. The tapered NF typically has a circular cross-section [31] 
but can also be made to have an elliptical cross section [34], as shown in Fig. 4(b). The elliptical NF can maintain a linear state of polarization and can be made by removing parts of the cladding of an SMF using a femtosecond laser before tapering. An SCF may be regarded as an NF core hanging at the center of the fiber by nanometer thin structs. SCF gas cells have been made by inflating selected air-holes of a photonic crystal fiber (PCF), and different core shapes can be made by inflating different air-holes. Figure 5 shows two examples of the SCFs made by inflating three and four of the inner most air-holes of a large mode area PCF from NKT Photonics [12]. The diameter of the inscribed core can be as small as $900 \mathrm{~nm}$ with tapered waist region up to 5 centimeters. The thin core of the SCF is naturally protected from external contamination and seamlessly integrated to the pristine PCF, which can be further spliced to SMFs with low loss [35]. SCFs with longer length may be directly drawn from a fiber drawing tower [9], although it may be challenging to splice a nanoscale-core SCF to a standard SMF with low loss.

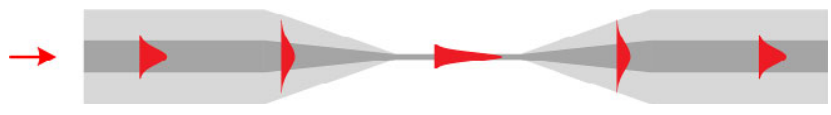

(a)

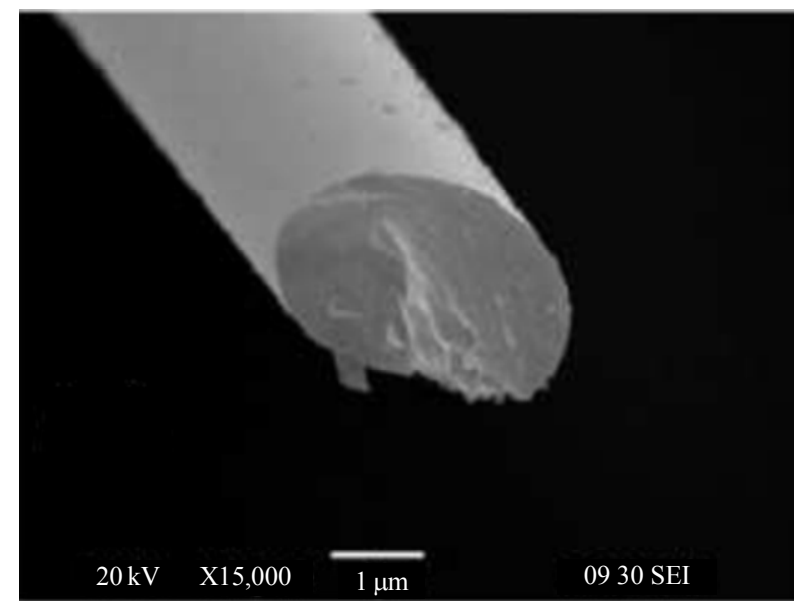

(b)

Fig. 4 NF gas cells: (a) mode field in a tapered NF and (b) an NF with elliptical cross-section [34].

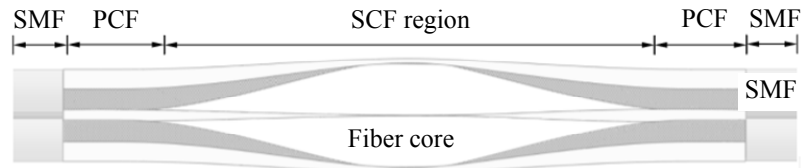

(a)
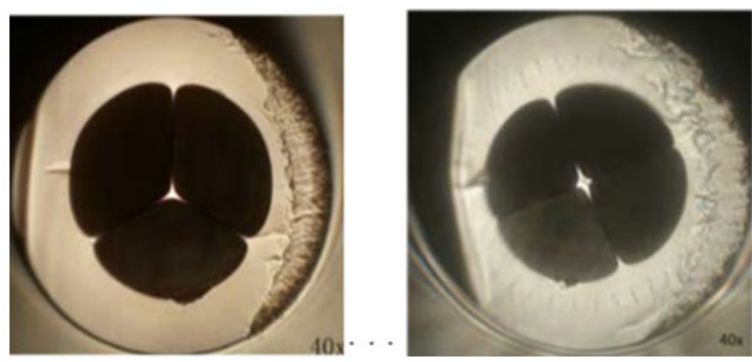

(b)

Fig. 5 SCF gas cells: (a) an SCF microcell made by inflating selected air-holes of a PCF and (b) cross-sections of two SCF microcells [32].

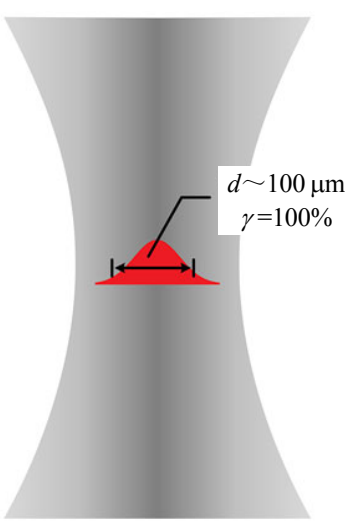

(a)

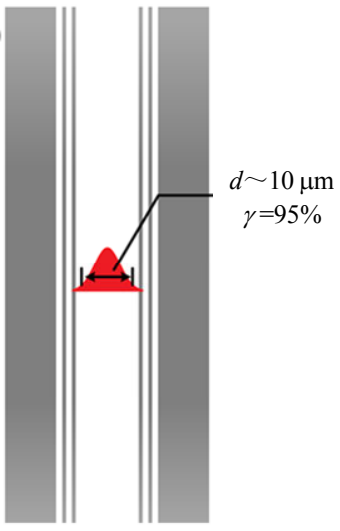

(b)

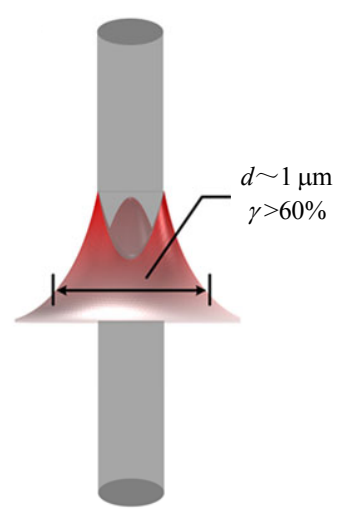

(c)
Fig. 6 Comparing open path, HCF, and NF gas cells [36].

To compare different gas cells, we may define an effective interaction length $\gamma L$ and a non-linear parameter $\gamma L / A$. Here, $L$ is the length of the MNOF, $\gamma$ is the fraction of mode power in air, and $A$ is the 
mode field area. For linear direct absorption spectroscopy, the signal is proportion to $\gamma L$ and an HCF gas cell may be used to achieve high sensitivity since it can be made sufficiently long without a significant increase in size. For nonlinear interaction such as stimulated Raman scattering, the signal is proportional to $\gamma L / A$. Compared with the free-space gas cell shown in Fig. 6(a), the HCF gas cell in Fig. 6(b) has a smaller mode field area $A$ extending over a much longer distance, which enhances the nonlinear interaction and hence enables higher detection sensitivity. The NF gas cell shown in Fig. 6(c) has an even smaller mode field area, i.e., $\sim 100$ times smaller than that of the HCF and $\sim 10^{4}$ times smaller than that of the free space beam, which would enhance enormously the nonlinear interaction per unit length. However, the length of an NF taper-drawn from an SMF is typically limited to less than 10 centimeters, due to the practical limitation of the current flame-brushing tapering systems.

\section{Direction absorption sensors}

Direct absorption optical sensor is based on Beer-Lambert Law:

$$
I_{\text {out }}=I_{0}(\lambda) \exp [-\alpha(\lambda) C \gamma L]
$$

where $C$ is the gas concentration, $\alpha(\lambda)$ the absorption coefficient, and $I_{0}(\lambda)$ is the light intensity without gas absorption. The use of the HCF enables compact all-fiber gas cells with longer effective absorption distance $\gamma L$ and hence potentially higher sensitivity. However, direct absorption sensors with PBG-HCF gas cells in the near infrared only demonstrated noise equivalent concentration (NEC) of a few parts per million (ppm) in volume fraction for gases with relatively strong absorption (e.g., $\mathrm{C}_{2} \mathrm{H}_{2}, \mathrm{NH}_{3}, \mathrm{CH}_{4}$ ) [20, 21, 37-39]. The performance was found primarily limited by multi-path interference, since a PBG-HCF typically supports multiple core and cladding modes for short lengths of fibers. An AR-HCF would support a smaller number of modes $[6,17]$ and could achieve better performance. Attempt has been made to develop single mode single polarization HCFs [40], which could in principle minimize the mode interference noise and enable higher sensitivity. With multiple micro-channels fabricated along a few meters of PBG-HCF, ppm level gas detection with response time from 3 seconds to 60 seconds has been demonstrated $[27,28]$. By using short lengths (e.g., $5 \mathrm{~cm}$ to $10 \mathrm{~cm}$ ) of PBG-HCF sandwiched between single mode fibers with mirrored ends, resonating Fabry-Perot gas cells with an effective optical length of several meters were demonstrated and achieved ppm level detection limit [41].

\section{Photothermal interferometry gas sensors}

Photothermal spectroscopy detects the heat generated due to light absorption. A pump-probe configuration is typically used, as shown in Fig.7. A pump laser beam is modulated in intensity and/or wavelength and tuned to an absorption line of a target gas, pump absorption results in periodic heating, which modulates the temperature, pressure, and density, and hence the refractive index distribution of gas material in the hollow-core. The probe wavelength is away from gas absorption and its accumulated phase is modulated [42, 43].

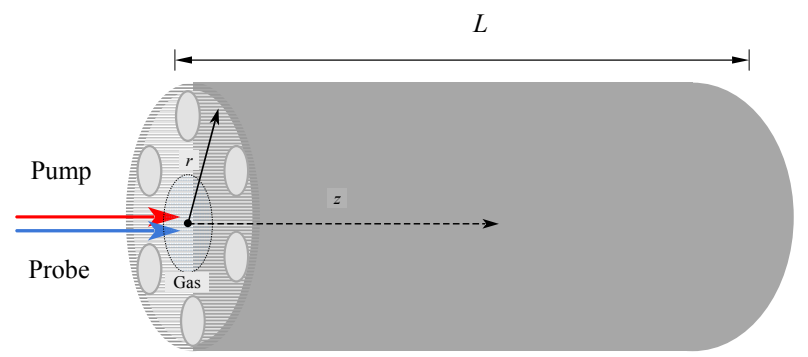

Fig. 7 Photothermal spectroscopy in a gas filled HCF. Pump and probe propagate in the HCF and interact with gas in the hollow-core.

Assuming that the pump is propagating in the fundamental $\mathrm{LP}_{01}$ mode, its intensity profile may be approximated by Gaussian distribution with the highest intensity at the core center and the intensity is reduced at the core-cladding boundary. Pump absorption results in higher temperature at the core center, which reduces the refractive index of gas 
material at the center and modulates the phase of the probe. The photothermal phase modulation is proportional to the overlap integral of the probe mode field with the induced refractive index distribution, and the amplitude of phase modulation per unit length along the HCF may be expressed as [43]

$$
\frac{\mathrm{d} \phi_{01}(z)}{\mathrm{d} z}=k \alpha(\lambda) C P_{\text {pump }}(z)
$$

where $P_{\text {pump }}(z)$ is the pump power at location $z$ along the HCF, $k$ is a constant depending on the frequency of pump modulation, which is determined by the HCF parameters, the wavelengths of the pump and probe, and the thermodynamic parameters of the gas material in the hollow-core, and $\phi_{01}(z)$ represents the phase of the $\mathrm{LP}_{01}$ mode. The total phase modulation may be determined by integrating (2) over the gas cell length $L$. For acetylene gas balanced with nitrogen in a hollow-core of $10 \mu \mathrm{m}$ in diameter, with both the pump and probe around $1550 \mathrm{~nm}$ and the pump wavelength aligned to the $\mathrm{P}(9)$ absorption line of acetylene at $1530.37 \mathrm{~nm}$, the phase modulation coefficient is on the order of $10^{-6} \mathrm{rad} /(\mathrm{mW} \cdot \mathrm{m} \cdot \mathrm{ppm})$ for modulation frequency up to $\sim 1 \mathrm{MHz}[44,45]$. For an HCF with a larger core diameter, the phase modulation is larger at low frequency but drops faster with increasing pump modulation frequency [46], as shown in Fig. 8 .

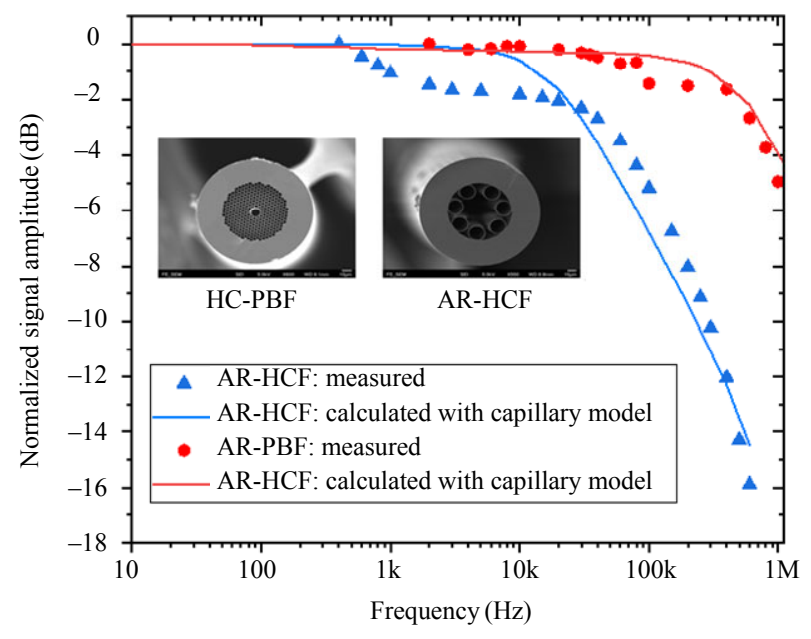

Fig. 8 Frequency response of photothermal phase modulation for two HCFs with different hollow-core diameters. The solid lines are the simulation results, while the triangles and circles are the measured results [46].
The photothermal phase modulation may be detected by various interferometer configurations. The first demonstration used a Mach-Zehnder probe interferometer (MZI) with the HCF gas cell placed in one arm of the interferometer [43]. By using servo control to stabilize the MZI at quadrature, the photothermal phase modulation was linearly converted to intensity modulation at the interferometer output. The wavelength of the pump was modulated sinusoidally and ramped slowly across the $\mathrm{P}(9)$ absorption line of acetylene around $1530.37 \mathrm{~nm}$. The second harmonic signal of the modulation frequency was detected for different concentrations of acetylene in nitrogen, as shown in Fig. 9(a). The NEC was estimated to be 2 parts per billion (ppb) with a 10-meter-long PBG-HCF gas cell. This is three orders of magnitude better than the direction absorption HCF sensors discussed in Section 3. The amplitude of the second harmonic increases with gas concentration and follows approximately a linear relationship for acetylene concentration up to 1\%, as shown in Fig. 9(b), giving a dynamic range of nearly six orders of magnitude.

The MZI detects the single-path phase modulation through the HCF, and the optical pathlengths of the two interfering beams may be made approximately balanced so that the phase noise of the probe source would not significantly affect the performance of phase detection. However, environment disturbance on the SMFs as well as Kerr nonlinearity in the SMFs, which form parts of the MZI, could result in unwanted phase modulation and affect the stability of phase measurement.

Sagnac interferometer is a single fiber interferometer in which the two interfering beams propagate in the same fiber but opposite directions. Such a system detects the phase difference between two counter propagating waves in a fiber loop, in which the HCF gas cell is placed near one end of the loop fiber and the pump modulation frequency and the fiber loop length are selected in a coordinative manner to maximize the differential photothermal phase modulation. The optical pathlengths of the 


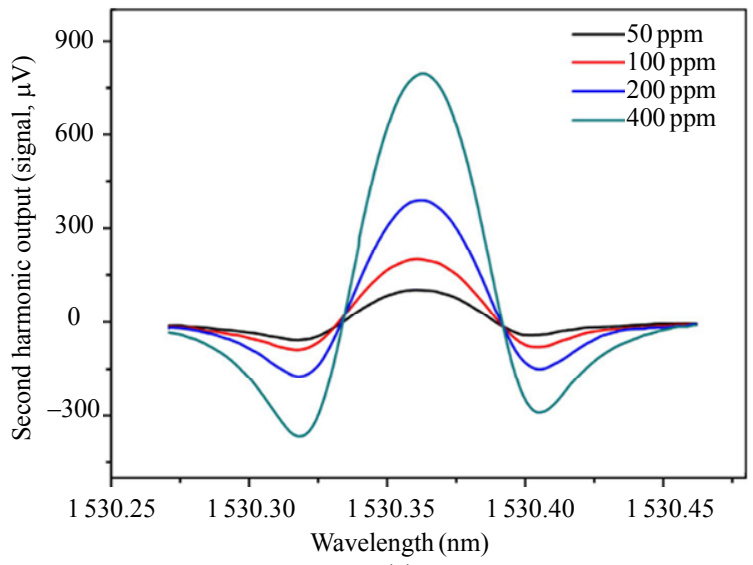

(a)

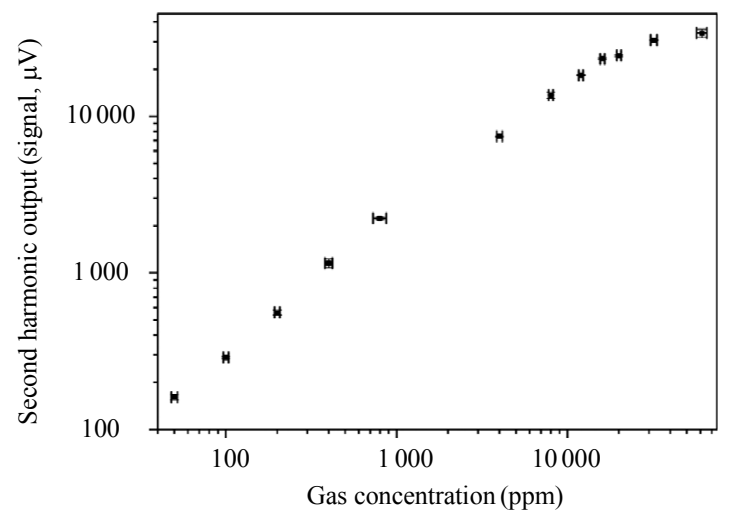

(b)

Fig. 9 Results of the first photothermal gas detection experiment with a HCF: (a) second harmonic output for different gas concentrations and (b) results of dynamic range test [43].

interfering beams are always balanced, which minimizes the effect of source phase noise and even allows the use of a broadband source to reduce the effect of multi-path interference and coherent backscatter. The single fiber configuration is more robust than the MZI since the two counterpropagating beams are affected similarly by the environment and the phase difference is relatively insensitive to external disturbance. Quadrature operation may be achieved passively by using a $3 \times 3$ loop coupler [45], as shown in Fig. 10(a), or using a frequency shifter inserted asymmetrically in the Sagnac loop [47]. With the Sagnac system shown in Fig. 10(a), detection of acetylene with NEC of $356 \mathrm{ppb}$ with 0.62-m-long PBG-HCF was demonstrated and the system has good stability with signal fluctuation of $<0.87 \mathrm{~dB}$ over a period of 6 hours [48], as shown in Fig. 10(b).

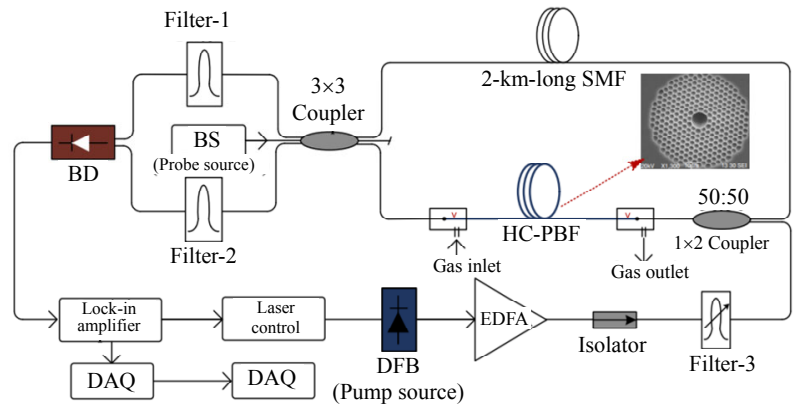

(a)

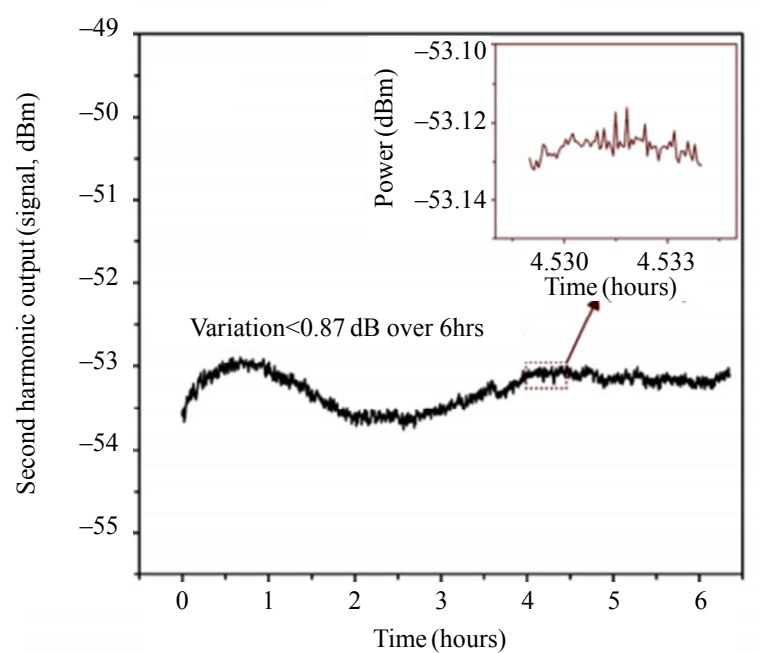

(b)

Fig. 10 Photothermal gas detection with a Sagnac interferometer to detect the phase difference between the counter propagating beams: (a) experimental setup. Output from the balanced detector (BD) contains the information of gas concentration; (b) results of stability test [48]. DFB laser: distributed feedback laser; EDFA: erbium doped fiber amplifier; BS: broadband source; DAQ: data acquisition card.

A simpler and more compact configuration is the single fiber reflective Fabry-Perot interferometer (FPI) [49]. A low-finesse FPI was formed naturally by the $4 \%$ reflections at the joints between the HCF and the SMF pigtails, as shown in Fig. 11(a). By using servo control to tune the cavity length or the wavelength of the probe laser, the FPI can be locked at a quadrature point at which the photothermal phase modulation is converted to intensity modulation in the FPI output. With a 5-cm-long AR-HCF and a pump power of $125 \mathrm{~mW}$ delivered into the HCF, NEC of $30 \mathrm{ppb}$ with $1 \mathrm{~s}$ lock-in time constant was achieved with detection system shown in Fig. 11(b). The dynamic range of the sensor was over 6 orders of magnitude. Allan deviation analysis showed that the NEC of $2.3 \mathrm{ppb}$ can be achieved 
with an integration time of $670 \mathrm{~s}$. Gas filling into the HCF can be achieved via the HCF/SMF joints and, because of the short length of the HCF, the response time as short as $52 \mathrm{~s}$ has been demonstrated.

In the FPI configuration, the phase difference between the interfering beams is twice that of the single pass phase modulation through the HCF and is relatively insensitive to environmental disturbance acting on the SMF transmission fiber. For a short length of HCF, the reflected probe beams come back into the SMF at approximately the same time (e.g., less than $1 \mathrm{~ns}$ difference for $15 \mathrm{~cm}$ long HCF); hence, low frequency disturbance on the SMF would affect the interfering beams similarly and has little effect on the phase difference. The Kerr nonlinearity of the SMF would have no effect on the differential phase modulation while the Kerr nonlinear coefficient of the HCF is two to three orders of magnitude smaller than the SMF.

However, the FPI is intrinsically an unbalanced interferometer and the optical path difference between the reflected beams is twice of the HCF length. Hence, the phase noise of the probe source will be converted into intensity noise at the FPI output, which could exceed other noise contributions if the source linewidth is not sufficiently narrow and/or if the HCF is too long.

In the reflective FPI, the probe light accesses the gas cell from one end of the HCF, leaving the other end free. This provides the flexibility of delivering the pump beam into the HCF, from the opposite end, with a fiber suitable for the pump wavelength (e.g., ultraviolet or infrared). This allows the use of a single HCF gas cell to detect different gas species with substantially different absorption wavelengths, assuming the HCF gas cell could transmit both the pump and the probe wavelengths simultaneously. Some of the current AR-HCFs have very broad transmission bands and would be ideal for such applications.

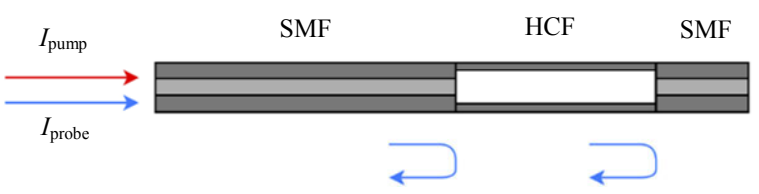

(a)

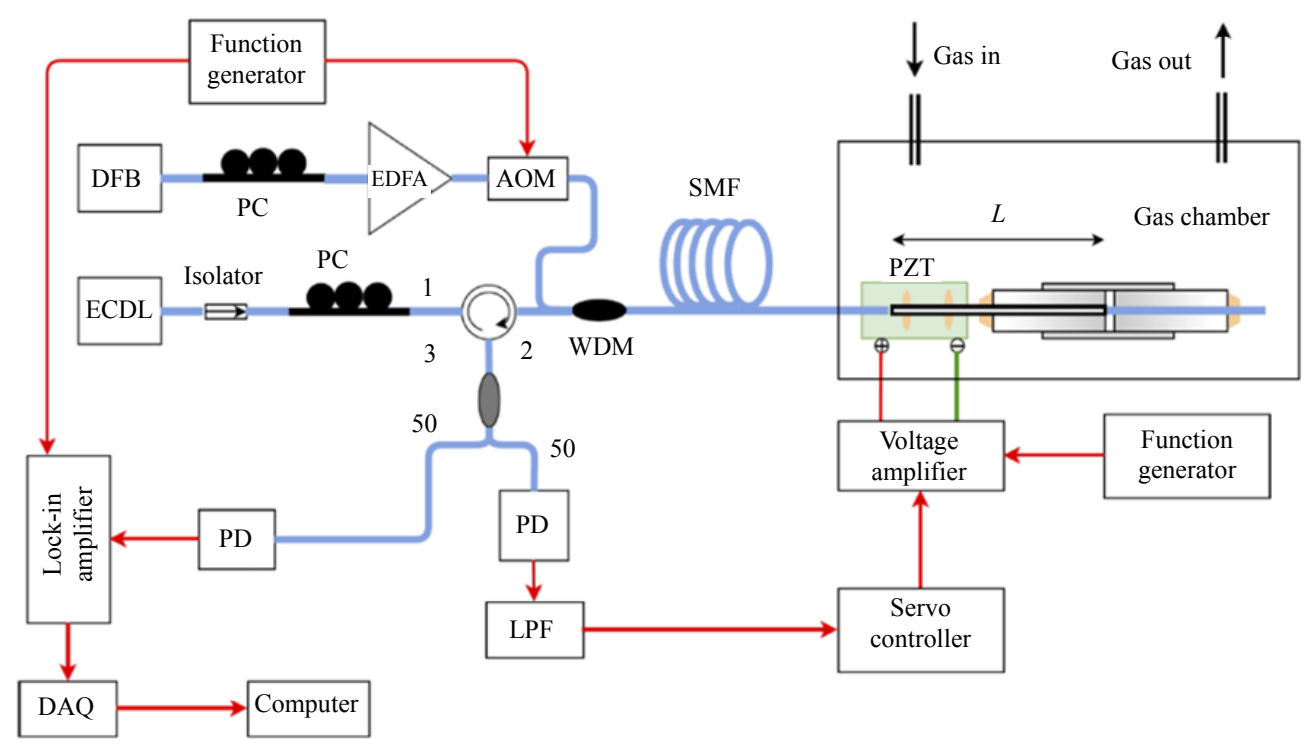

(b)

Fig. 11 Photothermal gas detection with a low-finesse FPI for phase detection: (a) schematic of the HCF FPI gas cell [46] and (b) a photothermal gas sensor with a low-finesse FPI for phase detection [46]. ECDL: external cavity diode laser; PC: polarization controller; LPF: low-pass filter; WDM: wavelength-division multiplexer; PZT: piezoelectric transducer; AOM: acoustic-optic modulator. 
With the FPI probe configuration operating at $1550 \mathrm{~nm}$ and different pump lasers from $760 \mathrm{~nm}$ to $3.5 \mu \mathrm{m}$, detection of oxygen, acetylene, methane, ethane, carbon monoxide, and carbon dioxide down to ppm to ppb level have been demonstrated [50-52], showing the potential wide range of applications of the technique.

From (2), it can be seen that the amplitude of photothermal phase modulation is proportional to pump power. By placing the HCF inside a high finesse passive Fabry-Perot cavity [53] or an active laser cavity [54], it is possible to enhance the pump power level to achieve higher phase modulation and hence potentially better gas detection sensitivity.

\section{Mode-phase-difference photothermal sensors}

The performance of photothermal gas sensors may be substantially improved by exploiting the unique modal properties of the optical fibers. By use of an HCF that supports dual transverse modes, i.e., the $\mathrm{LP}_{01}$ and $\mathrm{LP}_{11}$ modes, and detecting the photothermally induced phase difference between the two modes, we have demonstrated ultra-sensitive gas sensors down to parts per trillion (ppt) level [29]. Figure 12 shows the basic principle of mode-phase-difference photothermal spectroscopy. The pump beam is launched into the fundamental $\mathrm{LP}_{01}$ mode that has an approximately Gaussian intensity distribution in the fiber cross-section. The probe beam propagates in both $\mathrm{LP}_{01}$ and $\mathrm{LP}_{11}$ modes, which can be achieved by fabricating a long period grating (LPG) on the HCF to selectively couple a fraction of the probe $\mathrm{LP}_{01}$ mode power into the $\mathrm{LP}_{11}$ mode [55]. With offset alignment at the output HCF-SMF joint to form a dual-mode interferometer, the differential phase modulation can be detected. As mentioned earlier, the refractive index distribution due to pump absorption follows an inverse Gaussian profile, with the largest negative refractive index change occurring at the center of the HCF. The mode fields of the probe $\mathrm{LP}_{01}$ and $\mathrm{LP}_{11}$ modes are different, one has an approximate Gaussian profile while the other has a two-lobe distribution, which overlap differently with the nonuniform refractive index distribution and result in unequal phase modulation for the two probe modes. The phase modulation for the $\mathrm{LP}_{01}$ is larger than that of the $\mathrm{LP}_{11}$ mode, and the differential phase modulation is sensitive to gas absorption in the hollow-core and may be expressed as [29]: (a)

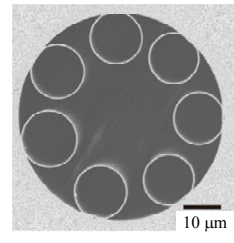

(b)

(c)
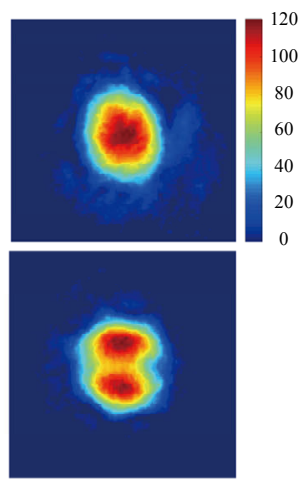

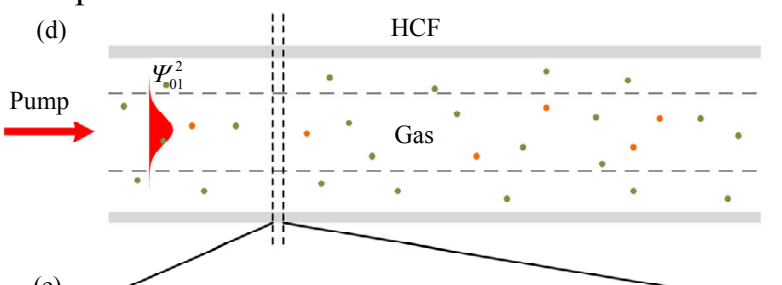

(e)

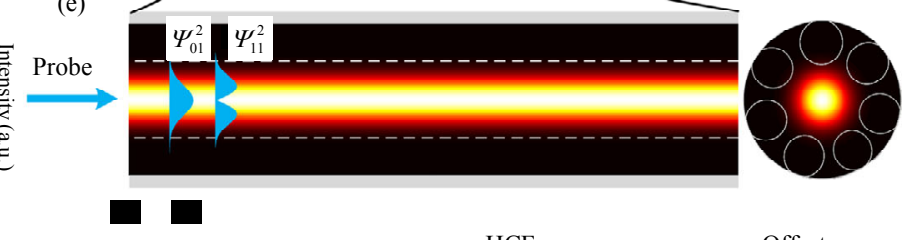

(f)

$\mathrm{HCF}$

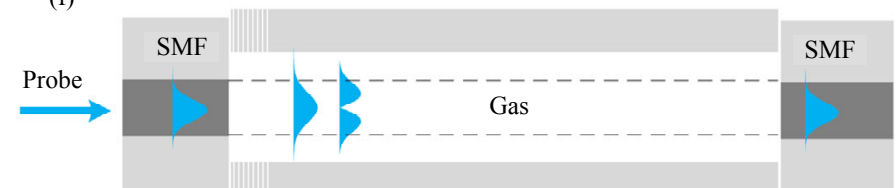

Fig. 12 Principle of mode-phase-difference photothermal spectroscopy [29]: (a) scanning electron microscope image of a two-mode AR-HCF, (b) and (c) measured near field image of LP $\mathrm{P}_{01}\left(\psi_{01}^{2}\right)$ and $\mathrm{LP}_{11}\left(\psi_{11}^{2}\right)$ guiding modes of the AR-HCF, (d) pump propagating in the $\mathrm{LP}_{01}$ mode, (e) refractive index change (yellow-red) due to pump absorption of gas molecules and the field distributions of the probe $\mathrm{LP}_{01}$ and $\mathrm{LP}_{11}$ mode (blue), and (f) practical implementation of a dual-mode interferometer with an LPG near the input end of the HCF and offset alignment at the output HCF-SMF joint. 


$$
\frac{\mathrm{d} \Delta \phi(z)}{\mathrm{d} z}=\frac{\mathrm{d}\left[\phi_{01}(z)-\phi_{11}(z)\right]}{\mathrm{d} z}=k^{*} \alpha(\lambda) C P_{\text {pump }}(z)
$$

where $k^{*}$ is the differential sensitivity coefficient and is about $20 \%$ of $k$ in (2). However, since the two probe modes propagate along the same $\mathrm{HCF}$, the sensitivity of the differential phase $\Delta \phi$ to external perturbation is much smaller than that the fundamental mode phase $\Delta \phi_{01}$, and ratio is $\sim\left(n_{01}-n_{11}\right) / n_{01}$, which is in the range of $10^{-2}$ to $10^{-3}$. This enhances the signal to noise ratio significantly and enables extremely sensitive photothermal gas sensors.

\section{Photoacoustic sensors}

HCFs may be used as highly efficient photoacoustic cells for gas detection based on photoacoustic spectroscopy. A wavelength or intensity modulated pump laser tuned to a gas absorption line generates acoustic wave via the photoacoustic effect. If the pump modulation frequency matches that of an acoustic mode of the gas filled HCF, the acoustic wave is resonantly amplified, which modulates the phase of a probe mode propagating in the HCF. A gas filled HCF may support two types of acoustic mode: the air modes that account for the density change of gas in the hollow core and the mechanical modes that are the displacements of the silica microstructure. Similar to the photothermal spectroscopy, the acoustic modes may be probed by detecting the phase modulation of the fundamental $\mathrm{LP}_{01}$ mode with one of the laser interferometer configurations described in Section 4 or the differential phase modulation between the $\mathrm{LP}_{01}$ and $\mathrm{LP}_{11}$ modes with the dual-mode fiber interferometer in Section 5. Figure 13 shows the detected differential phase modulation as a function of pump modulation frequency for a 70-cm-long AR-HCF filled with $106 \mathrm{ppm}$ of acetylene in nitrogen at 1 bar, in which the pump laser wavelength is tuned to center of the $\mathrm{P}(13)$ absorption line of acetylene around $1532.8 \mathrm{~nm}$. The mechanical modes of the capillaries (capillary resonance, $w_{1}$ ) and the air mode (radial resonance of gas, $\left.R_{02}\right)$ are clearly visible. The capillary resonances may be used to characterize the fiber microstructure. With the pump modulation frequency to a particular capillary resonance and the tuned pump laser wavelength across a gas absorption line, high sensitivity gas detection may be achieved.

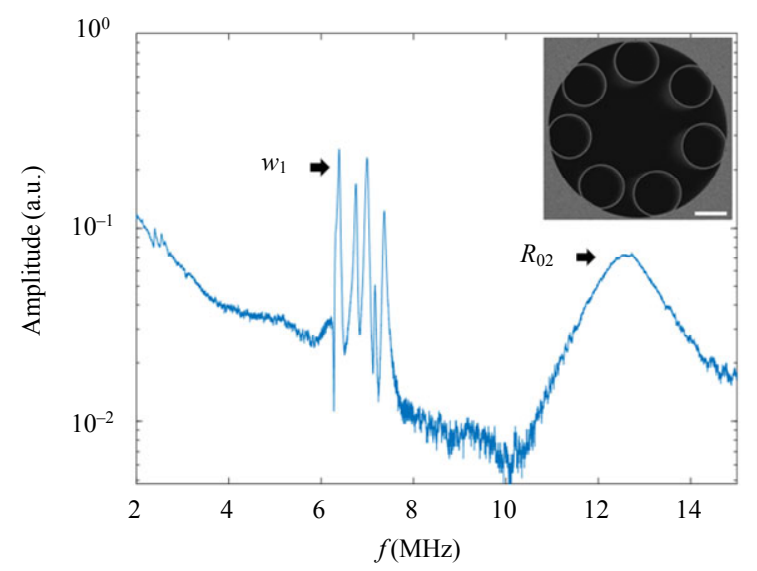

Fig. 13 Photoacoustic spectroscopy of a 70-cm-long AR-HCF filled with $106 \mathrm{ppm}$ of acetylene in nitrogen at 1 bar. The white bar in the SEM image is 20 micrometers.

\section{Raman sensors}

Raman spectroscopy can detect Raman-active gases that do not necessarily have an absorption line in the wavelength range of interest. However, the gas Raman signal in a free space system is extremely weak, limiting the usefulness of the technique for high sensitivity gas detection. MNOFs provide a compact platform for stronger light-gas interaction over a longer distance, which enhances Raman signal significantly and enables gas sensors with higher sensitivity. Earlier works on fiber Raman gas sensors operate in the visible and use HCF gas cells in combination with bulk optical components [56-59]. More recently, all-fiber gas sensors using stimulated Raman scattering (SRS) with HCFs as well as NFs have been demonstrated [60-62]. The SRS sensors have achieved ppm level NEC for the detection of hydrogen, which will be discussed in this section.

\subsection{HCF SRS sensors}

SRS involves interaction of gas molecules with 
two laser beams, a higher power pump with frequency $\omega_{\text {pump }}$ and a lower power probe (Stokes) with frequency $\omega_{\text {probe. }}$ When the frequency difference $\Delta \omega_{\text {diff }}=\omega_{\text {pump }}-\omega_{\text {probe }}$ matches a molecular Raman transition $\omega_{0}$, as shown in Fig. 14(a), energy from the pump will be transferred to the probe, resulting amplification of the probe and depletion of the pump. In the meantime, refractive index (RI) change (dispersion) is also induced in the vicinity of Raman transition, as shown in Fig. 14(b). The magnitudes of gain and dispersion are both proportional to pump optical intensity $I_{\text {pump }}$ (instead of optical power) and hence, a smaller mode field area would enhance the Raman gain and dispersion signals.

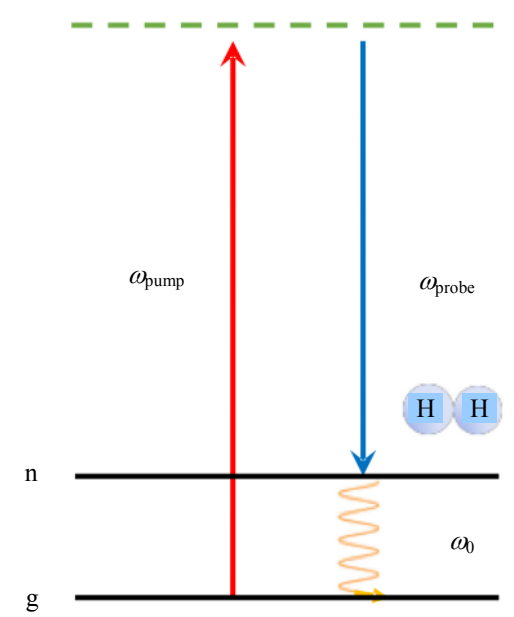

(a)
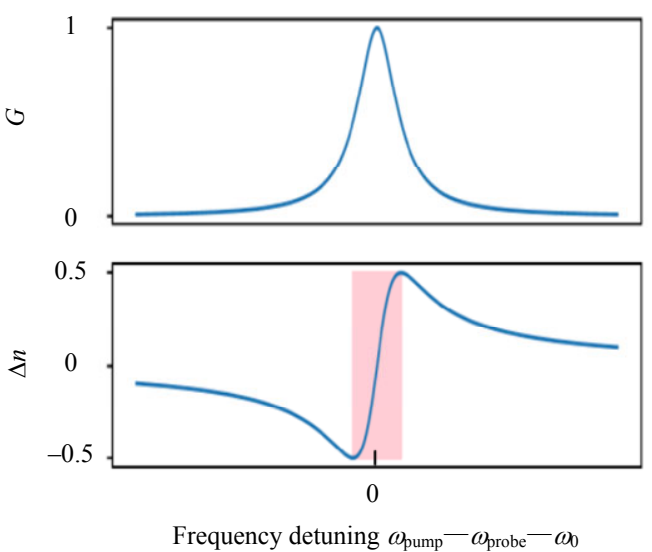

(b)

Fig. 14 SRS of hydrogen: (a) energy level diagram showing the SRS process. g: ground state; $\mathrm{n}$ : rotational state; the dotted line: intermediate state; (b) gain and dispersion around a Raman transition [62].
Stimulated Raman gain (SRG) spectroscopy detects the Raman amplification of a probe beam near a Raman transition. The SRG experienced by the probe beam may be expressed as $[61,63]$

$$
G\left(\omega_{\text {probe }}\right)=\frac{g_{0} I_{\text {pump }} L}{1+\delta^{2}}
$$

where $\delta=2\left(\omega_{0}-\Delta \omega_{\text {diff }}\right) / \Gamma_{R}$ is a linewidth-normalized frequency detuning parameter, $L$ is the length of $\mathrm{HCF}$, and $g_{0}$ is the Raman gain that is proportional to gas concentration $C$.

All-fiber hydrogen detection based on SRG spectroscopy was firstly demonstrated using the setup shown in Fig. 15. The wavelengths of the pump and Stokes beams are $\sim 1532 \mathrm{~nm}$ and $\sim 1620 \mathrm{~nm}$, respectively, which matches the $S_{0}(0)$ rotational Raman transition of hydrogen with Raman shift $\omega_{0}=354.36 \mathrm{~cm}^{-1}$. The probe wavelength was fixed while the pump wavelength was modulated sinusoidally at $\sim 50 \mathrm{kHz}$ and tuned slowly across the Raman transition. The second harmonic signal at $\sim 100 \mathrm{kHz}$ was detected and found increasing linearly with hydrogen concentration. The second harmonic was also increasing approximately linearly with pump power while the noise level remained relatively unchanged. With a pump power of $\sim 37 \mathrm{~mW}$ in a 15 -m-long HCF, the detection limit in terms of NEC for a signal to noise ratio of unity was $\sim 140 \mathrm{ppm}$ for $1 \mathrm{~s}$ lock-in time constant.

Stimulated Raman dispersion (SRD) spectroscopy detects the Raman-induced RI modulation near the Stokes wavelength. The induced RI modulation can be detected by measuring the phase modulation of the probe beam over length $L$ using an optical interferometer, which may be expressed as $[62,64]$ :

$$
\Delta \phi(\delta)=\frac{2 \pi}{\lambda_{\text {probe }}} \Delta n\left(\omega_{\text {probe }}\right) L=\left(\frac{\delta}{1+\delta^{2}}\right) \Delta \phi_{0}
$$

where $\Delta \phi_{0}=g_{0} I_{\text {pump }} L / 2$ is the peak phase modulation at the Raman resonance.

SRS-induced dispersion in a hydrogen-filled HCF was detected with the MZI system shown in Fig. 16(a). The MZI was stabilized at quadrature by use of a servo-loop. A 7-m-long HCF was filled 
with $20 \%$ hydrogen balanced with nitrogen and placed in one arm of the interferometer. The pump beam was wavelength modulated and tuned around $1532 \mathrm{~nm}$ to generate time-varying dispersion, which modulated the phase of the probe at $1620 \mathrm{~nm}$. Figure 16(b) shows the second harmonic signal from the lock-in amplifier for $20 \%$ hydrogen in nitrogen for different pump power levels. With pump power of $\sim 100 \mathrm{~mW}$ in the hollow-core, the NEC was estimated to be $25 \mathrm{ppm}$ for $1 \mathrm{~s}$ lock-in time constant.

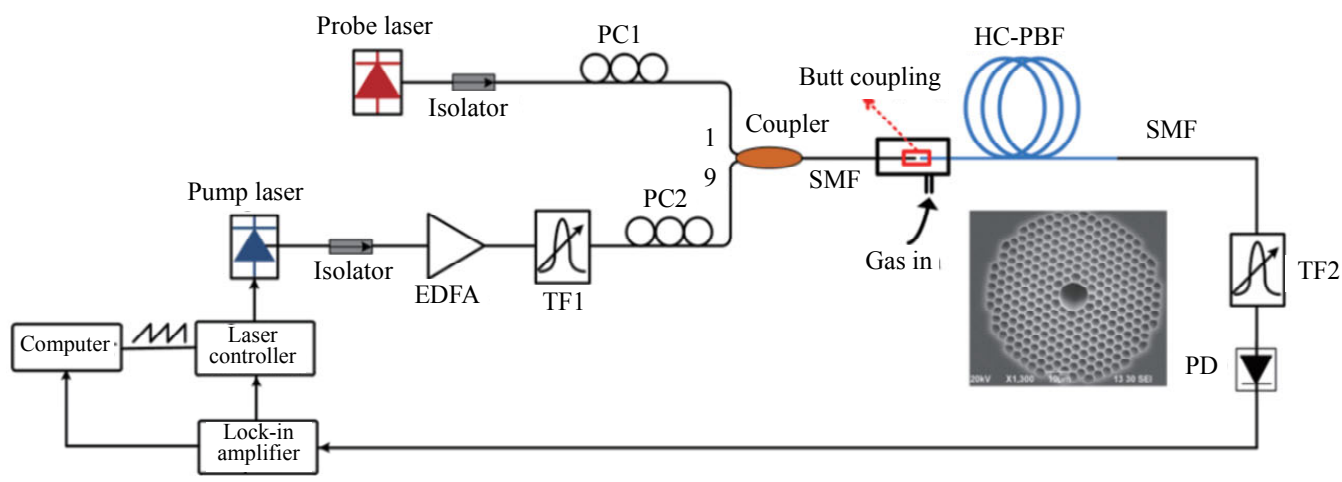

Fig. 15 Experimental setup of hydrogen detection with SRG spectroscopy[61]. TF: tunable filter.

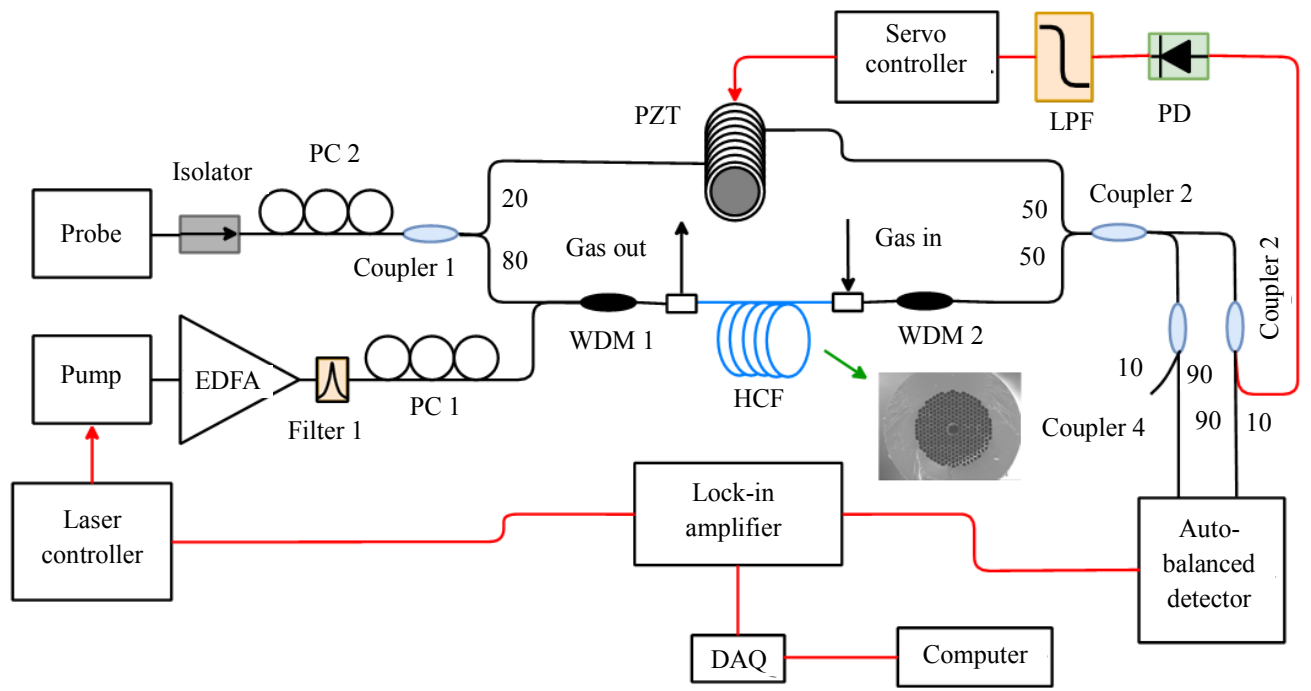

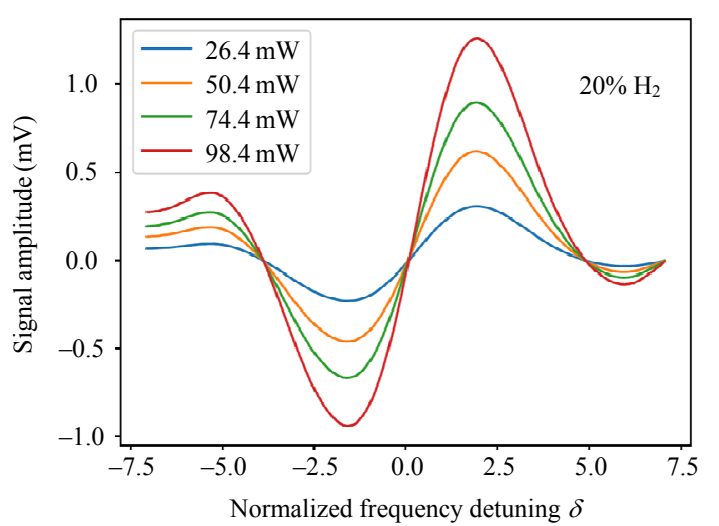

(b)

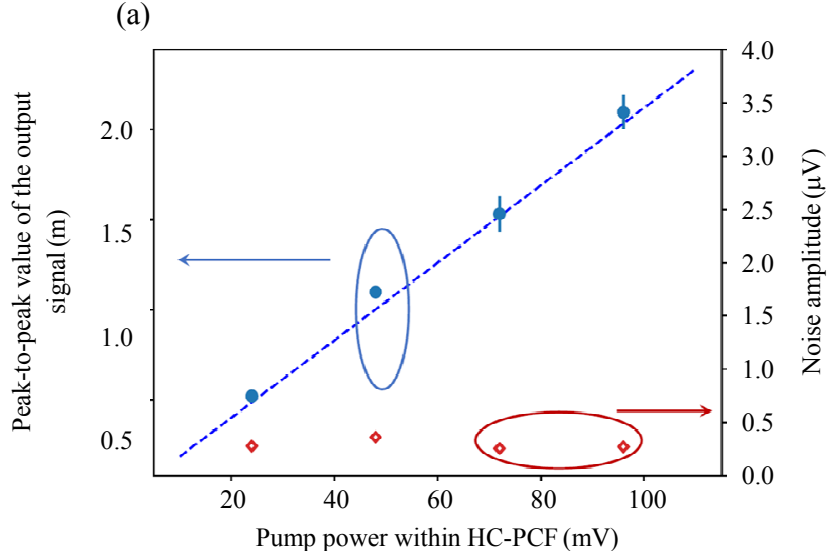

(c)

Fig. 16 Hydrogen detection with SRD spectroscopy: (a) setup for laser induced dispersion measurement, (b) second harmonic lock-in output when pump wavelength is tuned across the Raman transition for four different pump power levels and $20 \%$ hydrogen, and (c) peak-to-peak value of the second harmonic for $20 \%$ hydrogen and the standard deviation of noise as functions of pump power level [61]. 


\subsection{NF SRS sensors}

With the same setup as shown in Fig. 15 but the HCF replaced by an NF gas cell, SRG-based hydrogen detection was demonstrated [63]. The NF has a length of $\sim 5 \mathrm{~cm}$ and a diameter of $\sim 700 \mathrm{~nm}$ in the waist region. It was taper-drawn from a standard SMF28 fiber and spliced to SMFs with low loss. Figure 17(a) shows the measured second harmonic gain signals for different gas concentration as the pump wavelength was tuned across the $S_{0}(0)$ rotation Raman transition of hydrogen. The Raman signal increases linearly with hydrogen concentration from 0 to $50 \%$ hydrogen and nonlinearity appears from high hydrogen

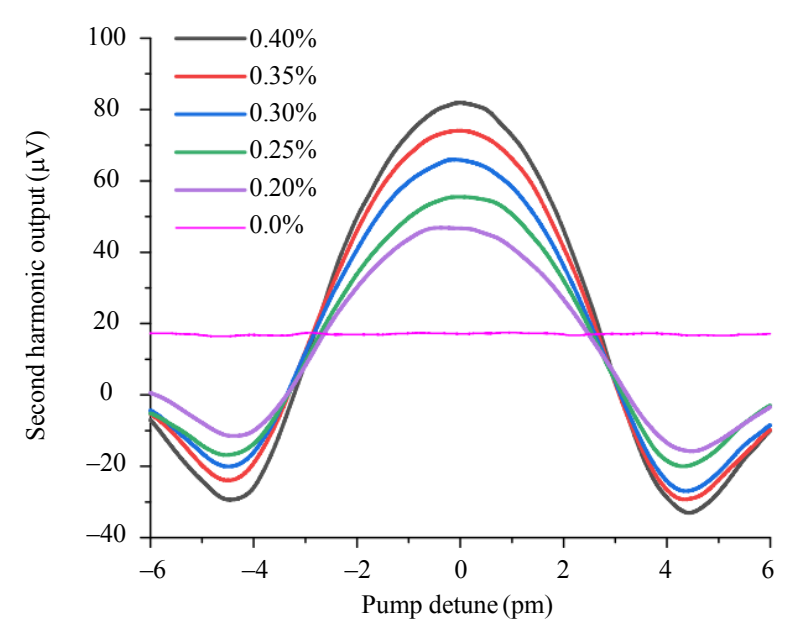

(a)

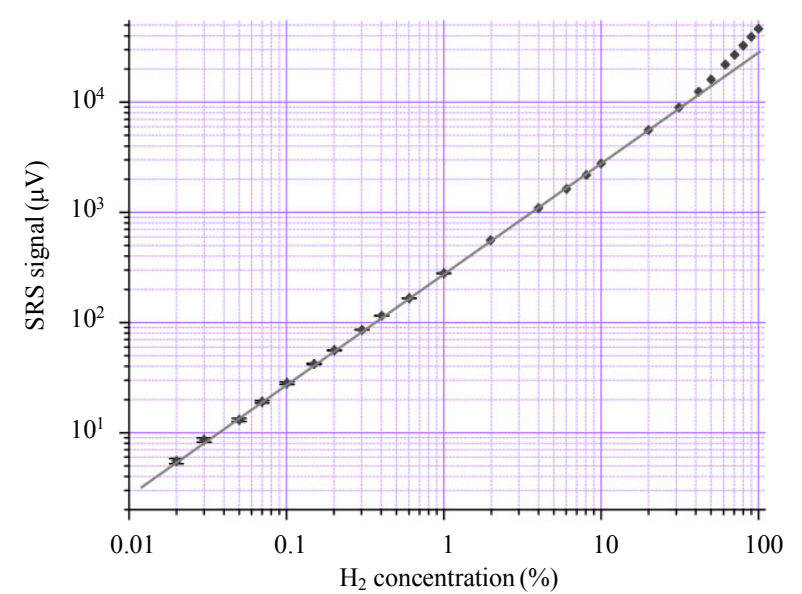

(b)

Fig. 17 Hydrogen detection based on SRG spectroscopy with an NF: (a) second harmonic output for pump tuning across the $S_{0}(0)$ Raman transition of hydrogen and (b) SRS signal for hydrogen concentration from 0 to $100 \%$ [62]. concentration, as shown in Fig. 17(b). For lock-in time constant of $10 \mathrm{~s}$ and pump power of $300 \mathrm{~mW}$, the NEC was estimated to be $8.6 \mathrm{ppm}$, giving a dynamic range of five orders of magnitude. The smaller mode field area and excellent mode quality of the tapered NF ensure higher Raman gain signal and minimal multi-path interference noise as compared with the HCF gas cells, which enables higher detection sensitivity. The exposed evanescent field allowed a faster response time of less than $10 \mathrm{~s}$.

\section{Distributed fiber gas sensors}

Distributed gas sensing would have many important applications such as pipeline gas leakage monitoring. The first distributed gas detection based on absorption spectroscopy was demonstrated with an optical time domain reflectometer (OTDR) and a pulsed DFB laser with its wavelength tuned to a gas absorption line acetylene [3]. The pulse duration was $\sim 10 \mathrm{~ns}$, giving a spatial resolution of $\sim 1 \mathrm{~m}$ over a length of 75 meters of PBG-HCF fiber.

Recently, distributed gas detection along a 200-m-long HCF was demonstrated with photothermal spectroscopy. The basic principle is shown in Fig. 18(a). The absorption of the modulated pump in a PBG-HCF generated distributed phase modulation along the fiber. The phase modulation frequency was determined by the pump modulation while its amplitude was proportional to local gas concentration, as given by (2). The backscattering in the PBG-HCF, primarily due to surface scattering of the hollow-core, was about an order of magnitude higher than Rayleigh scattering in a standard SMF around $1550 \mathrm{~nm}$. The backscattered waves carried the information of distributed photothermal phase modulation, which could be detected by a phase OTDR. Preliminary experiments with a dual-pulse phase OTDR achieved an NEC of $\sim 5 \mathrm{ppm}$ acetylene in nitrogen with a spatial resolution of $\sim 30 \mathrm{~m}$ [64]. 


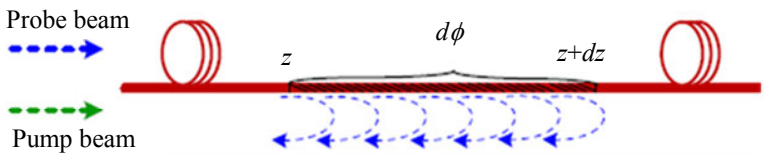

(a)

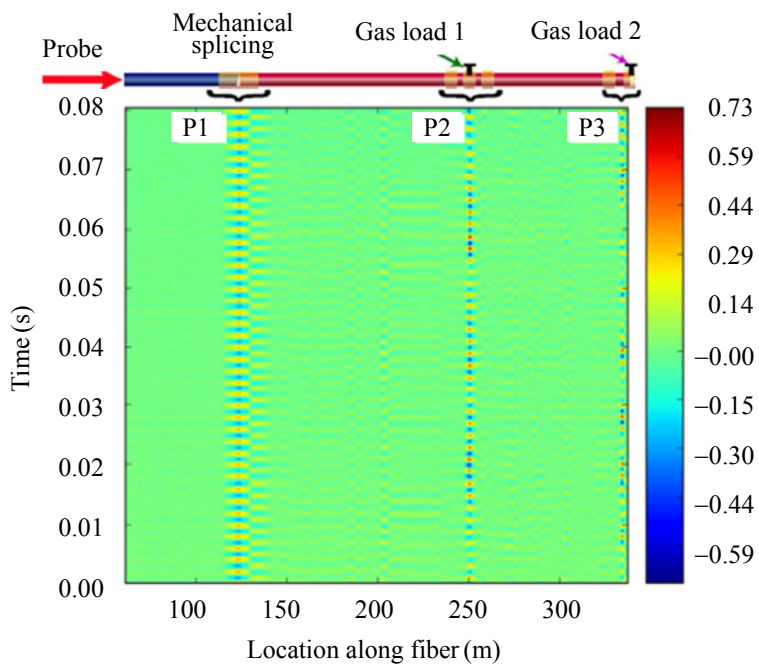

(b)

Fig. 18 Distributed gas detection with photothermal interferometry: (a) distributed gas detection with photothermal interferometry. Backscattered light is used to detect the distributed phase modulation along the fiber via a phase OTDR and (b) results of distributed gas detection along a 200 -m-long HCF. The upper part shows the fiber alignment and segments of sensing fiber. The sensing HCF (HC-1550-02) is from $130 \mathrm{~m}$ to $338 \mathrm{~m}$. P1 is the SMF/HCF joint and the larger signal at P1 is due to Kerr cross-phase modulation in the silica fiber. P2 and P3 are the two spatial locations ( $251 \mathrm{~m}$ and $335 \mathrm{~m}$ ) where the gas is loaded into the HCF [64].

More recently, distributed detection of hydrogen was performed based on SRG spectroscopy. A pulsed pump and a continuous-wave probe were used, as shown in Fig. 19(a) [65]. The frequency difference between the pump and probe was tuned to the $S_{0}(0)$ Raman transition of hydrogen. The probe beam was counter-propagating with the pump light and experienced Raman gain in the region filled with hydrogen when the pump pulse overlaps with the probe beam. The pump pulse entered the HCF from the SMF transmission fiber and the probe beam experienced firstly the SRG of the input SMF. The pump pulse then traveled down the HCF and the probe experienced SRG of hydrogen at a location where hydrogen molecules were present in the hollow-core. The pump pulse eventually reached the output SMF pigtail and the probe experienced the SRG of the output SMF.

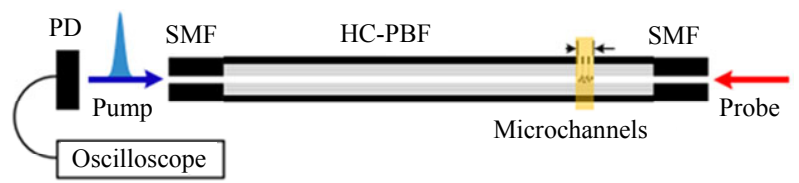

(a)

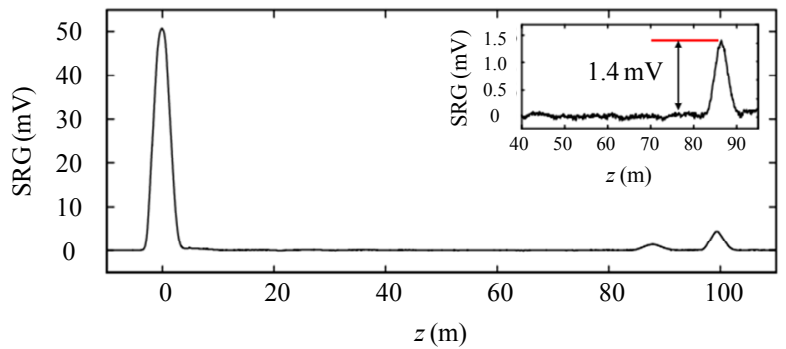

(b)

Fig. 19 Distributed detection of hydrogen along a HCF with SRG: (a) principle of distributed hydrogen sensing with SRG spectroscopy in HCF and (b) measured SRG trace along the 100-m-long HC-1550-02 HCF. The inset shows the SRG signal around $88 \mathrm{~m}$ where $4 \%$ hydrogen was present [65].

Figure 19(b) shows the measured distributed SRG signal of hydrogen over a 100-m-long PBG-HCF. Micro-channels were fabricated over a 2.2-m-long section around $88 \mathrm{~m}$ and were exposed to $4 \%$ of hydrogen at the pressure of $1 \mathrm{~atm}$. The two larger gain peaks at $0 \mathrm{~m}$ and $100 \mathrm{~m}$ were due to $\mathrm{SRG}$ of SMF pigtails. It marked the beginning and ending of the sensing HCF. The gain peak $88 \mathrm{~m}$ was due to the SRG of hydrogen. By comparing the SRG at $88 \mathrm{~m}$ with the standard deviation of fluctuation from $40 \mathrm{~m}$ to $80 \mathrm{~m}$, an NEC of $833 \mathrm{ppm}$ was estimated.

\section{Conclusions}

MONFs offer significantly advantages over the free space systems for linear and nonlinear spectroscopic gas sensing applications. The reduced mode field area, enhanced interaction length, and unique optical, thermal, and acoustic properties of the MNOFs enable gas sensors with significantly improved performance as well as creation of novel sensing concepts that are not possible with free space systems. MNOF gas sensors based on direct laser absorption, photothermal, photoacoustic 
Brillouin, and Raman spectroscopies have already demonstrated comparable or better performance over some of the complex free-space systems for a wide range of gas species. The compact size of the MNOF gas cells and possibility of seamless integrating them with fiber-based components and sub-systems would allow cost-effective interferometric detection to achieve superior performance.

Research is continuing in developing MNOF gas cells with better mode quality and polarization maintaining capability, in developing cavity enhanced system to achieve even better detection sensitivity and extending the applications of MNOF cells to the detection of trace substance in liquid [17].

\section{Acknowledgment}

The authors acknowledge the support of NSF of China (Grant No. 61827820), Hong Kong SAR Government GRF Grant (Grant No. PolyU152603/ 16E), the Local Innovative and Research Teams Project of Guangdong Pear River Talents Program (Grant No. 2019BT02X105), and the Hong Kong Polytechnic University (Grant Nos. P0034330 and 1-9B65).

Open Access This article is distributed under the terms of the Creative Commons Attribution 4.0 International License (http://creativecommons.org/licenses/by/4.0/), which permits unrestricted use, distribution, and reproduction in any medium, provided you give appropriate credit to the original author(s) and the source, provide a link to the Creative Commons license, and indicate if changes were made.

\section{Reference}

[1] J. Hodgkinson and R. P. Tatam, "Optical gas sensing: a review," Measurement Science and Technology, 2012, 24(1): 012004.

[2] B. Culshaw, G. Stewart, F. Dong, C. Tandy, and D. Moodie, "Fibre optic techniques for remote spectroscopic methane detection-from concept to system realisation," Sensors and Actuators B: Chemical, 1998, 51(1-3): 25-37.

[3] G. Stewart, W. Jin, and B. Culshaw, "Prospects for fibre-optic evanescent-field gas sensors using absorption in the near-infrared," Sensors and Actuators B: Chemical, 1997, 38(1-3): 42-47.

[4] W. Jin, H. L. Ho, Y. C. Cao, J. Ju, and L. F. Qi, "Gas detection with micro-and nano-engineered optical fibers," Optical Fiber Technology, 2013, 19(6): 741-759.

[5] P. Russell, "Photonic crystal fibers," Science, 2003, 299(5605): 358-362.

[6] P. Uebel, M. C. Günendi, M. H. Frosz, G. Ahmed, N. N. Edavalath, J. M. Ménard, et al., "Broadband robustly single-mode hollow-core PCF by resonant filtering of higher-order modes," Optics Letters, 2016, 41(9): 1961-1964.

[7] S. Gao, Y. Wang, W. Ding, D. Jiang, S. Gu, X. Zhang, et al., "Hollow-core conjoined-tube negativecurvature fibre with ultralow loss," Nature Communications, 2018, 9(1): 1-6.

[8] L. Tong and M. Sumetsky, Subwavelength and nanometer diameter optical fibers. Berlin: Springer Science \& Business Media, 2011.

[9] T. M. Monro, S. Warren-Smith, E. P. Schartner, A. François, S. Heng, H. Ebendorff-Heidepriem, et al., "Sensing with suspended-core optical fibers," Optical Fiber Technology, 2010, 16(6): 343-356.

[10]P. J. Roberts, F. Couny, H. Sabert, B. J. Mangan, D. P. Williams, L. Farr, et al., "Ultimate low loss of hollow-core photonic crystal fibres," Optics Express, 2005, 13(1): 236-244.

[11] T. P. Hansen, J. Broeng, C. Jakobsen, G. Vienne, H. R. Simonsen, M. D. Nielsen, et al., "Air-guiding photonic bandgap fibers: spectral properties, macrobending loss, and practical handling," Journal of Lightwave Technology, 2004, 22(1): 11-15.

[12] F. Benabid and P. J. Roberts, "Linear and nonlinear optical properties of hollow core photonic crystal fiber" Journal of Modern Optics, 2011, 58(2): 87-124.

[13] H. Sakr, Y. Chen, G. T. Jasion, T. D. Bradley, J. R. Hayes, H. C. H. Mulvad, et al., "Hollow core optical fibres with comparable attenuation to silica fibres between 600 and 1100 nm," Nature Communications, 2020, 11(1): 1-10.

[14]G. T. Jasion, T. D. Bradley, K. Harrington, H. Sakr, Y. Chen, E. N. Fokoua, et al., "Hollow core NANF with $0.28 \mathrm{~dB} / \mathrm{km}$ attenuation in the $\mathrm{C}$ and L bands," in Optical Fiber Communication Conference, United States, March 8-12, 2020: pp. Th4B. 4.

[15] J. M. Fini, "Microstructure fibres for optical sensing in gases and liquids," Measurement Science and Technology, 2004, 15(6): 1120.

[16] W. Jin, H. F. Xuan, and H. L. Ho, "Sensing with hollow-core photonic bandgap fibers," Measurement Science and Technology, 2010, 21(9): 094014.

[17]W. Jin, H. Bao, Y. Qi, Y. Zhao, P. Zhao, S. Gao, et al., "Micro nano-structured optical fiber laser spectroscopy," Acta Optica Sinica, 2021, 41(1): 
0130002

[18]F. Benabid, F. Couny, J. C. Knight, T. A. Birks, and P. S. J. Russell, "Compact, stable and efficient all-fibre gas cells using hollow-core photonic crystal fibres," Nature, 2005, 434(7032): 488-491.

[19] R. Thapa, K. Knabe, K. L. Corwin, and B. R. Washburn, "Arc fusion splicing of hollow-core photonic bandgap fibers for gas-filled fiber cells," Optics Express, 2006, 14(21): 9576-9583.

[20] Y. L. Hoo, W. Jin, H. L. Ho, J. Ju, and D. N. Wang, "Gas diffusion measurement using hollow-core photonic bandgap fiber," Sensors and Actuators B: Chemical, 2005, 105(2): 183-186.

[21] T. Ritari, J. Tuominen, H. Ludvigsen, J. C. Petersen, T. Sørensen, T. P. Hansen, et al., "Gas sensing using air-guiding photonic bandgap fibers," Optics Express, 2004, 12(17): 4080-4087.

[22] Y. L. Hoo, W. Jin, C. Shi, H. L. Ho, D. N. Wang, and S. C. Ruan, "Design and modeling of a photonic crystal fiber gas sensor," Applied Optics, 2003, 42(18): 3509-3515.

[23] R. M. Wynne, B. Barabadi, K. J. Creedon, and A. Ortega, "Sub-minute response time of a hollow-core photonic bandgap fiber gas sensor," Journal of Lightwave Technology, 2009, 27(11): 1590-1596.

[24] J. P. Parry, B. C. Griffiths, N. Gayraud, E. D. McNaghten, A. M. Parkes, W. N. MacPherson, et al., "Towards practical gas sensing with micro-structured fibres," Measurement Science and Technology, 2009, 20(7): 075301.

[25] C. M. B. Cordeiro, E. M. dos Santos, C. H. Brito Cruz, C. J. S. de Matos, and D. S. Ferreira, "Lateral access to the holes of photonic crystal fibers-selective filling and sensing applications," Optics Express, 2006, 14(18): 8403-8412.

[26] C. J. Hensley, D. H. Broaddus, C. B. Schaffer, and A. L. Gaeta, "Photonic band-gap fiber gas cell fabricated using femtosecond micromachining," Optics Express, 2007, 15(11): 6690-6695.

[27] Y. L. Hoo, S. Liu, H. L. Ho, and W. Jin, "Fast response microstructured optical fiber methane sensor with multiple side-openings," IEEE Photonics Technology Letters, 2010, 22(5): 296-298.

[28] F. Yang, W. Jin, Y. Lin, C, Wang, H. Lut, and Y. Tan, "Hollow-core microstructured optical fiber gas sensors," Journal of Lightwave Technology, 2016, 35(16): 3413-3424.

[29] P. Zhao, Y. Zhao, H. Bao, H. L. Ho, W. Jin, S. Fan, et al., "Mode-phase-difference photothermal spectroscopy for gas detection with an anti-resonant hollow-core optical fiber," Nature Communications, 2020, 11: 847.

[30] W. Belardi, "Design and properties of hollow antiresonant fibers for the visible and near infrared spectral range," Journal of Lightwave Technology, 2015, 33(21): 4497-4503.

[31] W. Jin, H. Xuan, C. Wang, W. Jin, and Y. Wang,
"Robust microfiber photonic microcells for sensor and device applications," Optics Express, 2014, 22(23): 28132-28141.

[32] C. Wang, W. Jin, J. Ma, Y. Wang, H. L. Ho, and X. Shi, "Suspended core photonic microcells for sensing and device applications," Optics Letters, 2013, 38(11): 1881-1883.

[33] T. A. Birks and Y. W. Li, "The shape of fiber tapers," Journal of Lightwave Technology, 1992, 10(4): 432-438.

[34] H. Xuan, J. Ju, and W. Jin, "Highly birefringent optical microfibers," Optics Express, 2010, 18(4): 3828-3839.

[35] L. Xiao, M. S. Demokan, W. Jin, Y. Wang, and C. L. Zhao, "Fusion splicing photonic crystal fibers and conventional single-mode fibers: microhole collapse effect," Journal of Lightwave Technology, 2007, 25(11): 3563-3574.

[36] Y. Qi, F. Yang, Y. Lin, W. Jin, and H. L. Ho, "Nanowaveguide enhanced photothermal interferometry spectroscopy," Journal of Lightwave Technology, 2017, 35(24): 5267-5275.

[37] L. Kornaszewski, N. Gayraud, J. M. Stone, W. N. MacPherson, A. K. George, J. C. Knight, et al., "Mid-infrared methane detection in a photonic bandgap fiber using a broadband optical parametric oscillator," Optics Express, 2007, 15(18): 11219-11224.

[38] A. M. Cubillas, J. Hald, and J. C. Petersen, "High resolution spectroscopy of ammonia in a hollow-core fiber," Optics Express, 2008, 16(6): 3976-3985.

[39] F. Yang, W. Jin, Y. Cao, H. L. Ho, and Y. Wang, "Towards high sensitivity gas detection with hollow-core photonic bandgap fibers," Optics Express, 2014, 22(20): 24894-24907.

[40] J. M. Fini, J. W. Nicholson, B. Mangan, L. Meng, R. S. Windeler, E. M. Monberg, et al., "Polarization maintaining single-mode low-loss hollow-core fibres," Nature Communications, 2014, 5: 5085.

[41] Y. Tan, W. Jin, F. Yang, Y. Qi, Co. Zhang, Y. Lin, et al., "Hollow-core fiber-based high finesse resonating cavity for high sensitivity gas detection," Journal of Lightwave Technology, 2017, 35(14): 2887-2893.

[42] S. E. Bialkowski, "Photothermal spectroscopy methods for chemical analysis," America: John Wiley \& Sons, 1996.

[43] W. Jin, Y. Cao, F. Yang, and H. L. Ho, "Ultra-sensitive all-fibre photothermal spectroscopy with large dynamic range," Nature Communications, 2015, 6: 6767.

[44] Y. Lin, W. Jin, F. Yang, Y. Tan, and H. L. Ho, "Performance optimization of hollow-core fiber photothermal gas sensors," Optics Letters, 2017, 42(22): 4712-4715. 
[45] Y. Lin, W. Jin, F. Yang, J. Ma, C. Wang, H. L. Ho, et al., "Pulsed photothermal interferometry for spectroscopic gas detection with hollow-core optical fibre," Scientific Reports, 2016, 6(1): 1-12.

[46] H. Bao, Y. Hong, W. Jin, H. L. Ho, C. Wang, S. Gao, et al., "Modeling and performance evaluation of in-line Fabry-Perot photothermal gas sensors with hollow-core optical fibers," Optics Express, 2020, 28(4): 5423-5435.

[47] J. Ma, Y. Yu, and W. Jin, "Demodulation of diaphragm based acoustic sensor using Sagnac interferometer with stable phase bias," Optics Express, 2015, 23(22): 29268-29278.

[48] Y. Lin, W. Jin, F. Yang, and C. Wang, "Highly sensitive and stable all-fiber photothermal spectroscopic gas sensor," in CLEO: Science and Innovations 2016, San Jose, California United States, June 5-10, 2016, pp. STu4H. 3.

[49] F. Yang, Y. Tan, W. Jin, Y. Lin, Y. Qi, and H. L. Ho, "Hollow-core fiber Fabry-Perot photothermal gas sensor," Optics Letters, 2016, 41(13): 3025-3028.

[50] C. Yao, Q. Wang, Y. Lin, W. Jin, L. Xiao, S. Gao, et al., "Photothermal CO detection in a hollow-core negative curvature fiber," Optics Letters, 2019, 44(16): 4048-4051.

[51] Y. Hong, H. Bao, W. Jin, S. Jiang, H. L. Ho, S. Gao, et al., "Oxygen gas sensing with photothermal spectroscopy in a hollow-core negative curvature fiber," Sensors, 2020, 20(21): 6084.

[52]F. Chen, S. Jiang, W. Jin, H. Bao, H. L. Ho, C. Wang, et al., "Ethane detection with mid-infrared hollow-core fiber photothermal spectroscopy," Optics Express, 2020, 28(25): 38115-38126.

[53] Y. Tan, W. Jin, F. Yang, Y. Jiang, and H. L. Ho, "Cavity-enhanced photothermal gas detection with a hollow fiber Fabry-Perot absorption cell," Journal of Lightwave Technology, 2019, 37(17): 4222-4228.

[54] Y. Zhao, W. Jin, Y. Lin, F. Yang, and H. L. Ho, "All-fiber gas sensor with intracavity photothermal spectroscopy," Optics Letters, 2018, 43(7): 1566-1569.

[55] P. Zhao, H. L. Ho, W. Jin, S. Fan, S. Gao, Y. Wang, et al., "Gas sensing with mode-phase-difference photothermal spectroscopy assisted by a long period grating in a dual-mode negative-curvature hollow-core optical fiber," Optics Letters, 2020, 45(20): 5660-5663.

[56] M. P. Buric, K. P. Chen, J. Falk, and S. D. Woodruff, "Enhanced spontaneous Raman scattering and gas composition analysis using a photonic crystal fiber," Applied Optics, 2008, 47(23): 4255-4261.

[57] J. L. Doménech and M. Cueto, "Sensitivity enhancement in high resolution stimulated Raman spectroscopy of gases with hollow-core photonic crystal fibers," Optics Letters, 2013, 38(20): 4074-4077.

[58] P. G. Westergaard, M. Lassen, and J. C. Petersen, "Differential high-resolution stimulated CW Raman spectroscopy of hydrogen in a hollow-core fiber," Optics Express, 2015, 23(12): 16320-16328.

[59] S. Hanf, T. Bögözi, R. Keiner, T. Frosch, and J. Popp, "Fast and highly sensitive fiber-enhanced Raman spectroscopic monitoring of molecular $\mathrm{H}_{2}$ and $\mathrm{CH}_{4}$ for point-of-care diagnosis of malabsorption disorders in exhaled human breath," Analytical Chemistry, 2015, 87(2): 982-988.

[60] F. Yang and W. Jin, “All-fiber hydrogen sensor based on stimulated Raman gain spectroscopy with a 1550 nm hollow-core fiber," in 2017 25th Optical Fiber Sensors Conference (OFS), Jeju, April 24-28, 2017, pp. $1-4$.

[61] H. Bao, W. Jin, Y. Miao, and H. L. Ho, "Laser-induced dispersion with stimulated Raman scattering in gas-filled optical fiber," Journal of Lightwave Technology, 2019, 37(9): 2120-2125.

[62] Y. Qi, Y. Zhao, H. Bao, W. Jin, and H. L. Ho, "Nanofiber enhanced stimulated Raman spectroscopy for ultra-fast, ultra-sensitive hydrogen detection with ultra-wide dynamic range," Optica, 2019, 6(5): 570-576.

[63] R. W. Boyd, Nonlinear optics. America: Academic Press, 2020.

[64] Y. Lin, F. Liu, X. He, W. Jin, M. Zhang, F. Yang, et al., "Distributed gas sensing with optical fibre photothermal interferometry," Optics Express, 2017, 25(25): 31568-31585.

[65] F. Yang, Y. Zhao, Y. Qi, Y. Z. Tan, H. L. Ho, and W. Jin, "Towards label-free distributed fiber hydrogen sensor with stimulated Raman spectroscopy," Optics Express, 2019, 27(9): 12869-12882. 